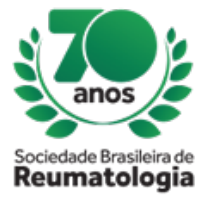

\title{
EPIDEMIOLOGICAL PROFILE OF PATIENTS USING BIOLOGICAL THERAPY ACCOMPANIED BY A RHEUMATOLOGY OUTPATIENT CLINIC IN FEIRA DE SANTANA-BA
}

Laura Sabrina de Almeida Fernandes (Universidade Estadual de Feira de Santana, Feira de Santana, BA, Brasil), Lucas dos Santos Silva (Universidade Estadual de Feira de Santana, Feira de Santana, BA, Brasil), Ana Teresa Amoedo (Universidade Estadual de Feira de Santana, Feira de Santana, BA, Brasil), Blenda Maria dos Santos Erdes (Universidade Estadual de Feira de Santana, Feira de Santana, BA, Brasil), Dyalle Costa e Silva (Universidade Estadual de Feira de Santana, Feira de Santana, BA, Brasil), Evelly Cerqueira de Amorim Paixão (Universidade Estadual de Feira de Santana, Feira de Santana, BA, Brasil), Filipe Mota Freitas (Universidade Estadual de Feira de Santana, Feira de Santana, BA, Brasil), João Mário Aguiar Abrantes Dourado (Universidade Estadual de Feira de Santana, Feira de Santana, BA, Brasil), Jordyr Matheus Magalhães Rocha (Universidade Estadual de Feira de Santana, Feira de Santana, BA, Brasil), Michele Karen Pereira Clementino (Universidade Estadual de Feira de Santana, Feira de Santana, BA, Brasil), Rosimary Alves da Silva (Universidade Estadual de Feira de Santana, Feira de Santana, BA, Brasil)

\section{BACKGROUND}

Over the past two decades, great strides have been made in understanding the immune system and in the pathogenesis of various diseases. This coincided with the development of biological therapies monoclonal antibodies and fusion proteins. The use of biological therapy for the treatment of autoimmune and rheumatologic diseases is expanding rapidly due to good efficacy and safety profiles. The objective of this study was to analyze the epidemiological profile of patients who use biological therapy in a rheumatology outpatient clinic in Feira de Santana - BA.

\section{MATERIALS AND METHODS}

This is a quantitative, descriptive, retrospective and documentary study based on medical records, carried out in a rheumatology outpatient clinic. A total of 366 medical records of patients attended from February 2008 to May 2019 were analyzed, and the medical records of patients who received or were abandoned at the end of the study period were excluded from the analysis. The instrument used to collect data from the medical record was a form that addressed the following aspects: identification, clinical data, personal history and medication in use, later the data were grouped in a database and examined by Epidata Entry software.

\section{RESULTS}

We included 33 patients in biological therapy, $9 \%$ of those followed by the outpatient clinic. The majority are female $(63.6 \%)$, single $(42.4 \%)$ and age ranged from $12-81$, averaging 46.5 years. The majority of the patients declared themselves mulatto $(42.4 \%)$, followed by white $(21.2 \%)$, ignored $(21.2 \%)$ and black (15.2\%). The most frequent occupations were: Retired (12.1\%), student (12.1\%), farmer (12.1\%), housewife (9.1\%). 39.4\% had Rheumatoid Arthritis (RA) as the main diagnosis, $24.2 \%$ ankylosing spondylitis and $21.2 \%$ spondyloarthritis (AE). The most used biological was Etanercept $(39.4 \%)$ in the treatment of RA $(53.8 \%)$, followed by Adalimumab (18.2\%) in RA (33.3\%) and AD (33.3\%) and by infliximab $(18.2 \%)$ in ankylosing spondylitis (66.7\%). $39.4 \%$ also use some DMARDs, of which $76.9 \%$ use Methotrexate. Only $6.1 \%$ of patients use hydroxychloroquine. The most frequent comorbidities were hypertension (30.3\%), diabetes mellitus (15.2\%) and infections (15.2\%). $39.4 \%$ of the patients were hospitalized and $42.4 \%$ underwent surgery, $12.1 \%$ were smokers, $15.2 \%$ were alcoholics, and $15.2 \%$ practiced physical exercises.

\section{CONCLUSION}


Biological therapy is restricted to a small proportion of rheumatic patients, and the majority are women, mulattos, single and in middle age. Despite the morbidities and limitations, the majority of patients maintain a labor activity that may have been made possible by the use of the therapy. 